\title{
Explosive technologies in transport construction
}

\author{
Nail Tuktamyshov ${ }^{1 *}$ [0000-0002-4679-0701] \\ ${ }^{1}$ Kazan State University of Architecture and Engineering, 420043, Zelenaya st., Kazan, Russia
}

\begin{abstract}
Performing different works (construction of road embankments, canals, tunnels, moving some soil mass in a given direction), as well as construction of various towers, power transmission supports, foundations for bridge supports, special pits for anchors are carried out by explosive technologies. The paper analyzes in detail a new method of determining the boundary of the explosion excavation. Its distinguishing features are the use of an impulse-hydrodynamic model for setting explosion problems and use of boundary problems of the theory of analytical functions. The new method is used in solving the problem of determining the boundary of the ejection excavation for the case of a buried charge. The results of calculations for the main geometric parameters of the explosion are given. The prospects for the development and application of the method are discussed.
\end{abstract}

Keywords:road embankments, canals, explosive technologies, cord charges, impulse, complex potential.

\section{Introduction}

The energy of an explosion is a special type of energy that can be easily metered, its transfer does not require stationary communications, and its ability to perform work eliminates the need for complex working machines. Its high concentration and enormous power characteristics have also determined the special field for the use of the energy of explosion where other energy sources are ineffective.

Explosive technologies have found wide application at development of mineral resources [1, 2], at consolidation of grounds [3, 4], they are used at reception of underground tanks [5, 6], in tests of building designs on impulse influence [7]. At manufacture of some works (building of road embankments, channels, foundation pits) there is a task of moving some weight of a ground in the set direction. Construction of various towers, power transmission towers, and foundations for bridge supports, special foundation pits for anchors and their concreting is carried out using explosive technologies. During excavation works related to the construction of road embankments, trenches and wells, so-called cord charges are used. As experiments show, the movement of the medium accompanying the explosion can be divided into two stages. The first, short-term, is characterized by the propagation of the stress wave and a relatively small increase in displacements and velocities of the particles. At this stage, reflections may occur and destruction may occur.

\footnotetext{
*Corresponding author: nail1954@gmail.com
} 
The first stage is associated with numerical modeling of detonation [8], in particular, with the grid modeling of detonation waves [9], with the study of shock waves [10,11], and also with the structure of shock waves $[12,13]$. A number of works are devoted to the analysis of borehole charge initiation [14], the influence of shock wave shapes on explosion parameters [15], and the construction of shock wave computational schemes in special cases [16-18].

The second stage is ballistic. There is either non-camouflage explosion or individual blocks are thrown away from the point of explosion. The study of the second stage is of interest not only in the construction, but also in the study of the formation of craters of celestial bodies [19-21]. At the end of the first stage, a velocity field "initial" for the ballistic stage is produced. At the first stage, the fields of stress and velocity are determined mainly by the inertial resistance of the medium, so the compressibility can be neglected. Since the pressure at the initial stage of the explosion is very high, a second assumption is made to consider the strength effects as secondary and to describe the state of the ball pressure tensor. All this makes it possible to assume that the medium is incompressible, that the medium is ideal (that is, there are no tangential stresses), and that deformations and displacements remain small. These assumptions make it possible to use the model of an ideal incompressible fluid to calculate the size, embankment, tunnel or trench when calculating the explosion for an ejection. M. A. Lavrentyev introduced an additional strength characteristic of the soil - critical velocity.

Built by Lavrentyev M. A., the model is called impulse-hydrodynamic and applied by V. M. Kuznetsov [22]. Due to the large magnitude of the explosive loads and the short duration of their action at the initial stage of the explosion medium can be considered an ideal incompressible liquid, then its movement is described by the equation.

$$
\frac{d \bar{v}}{d t}=-\nabla p / \rho
$$

or $\operatorname{div} \bar{v}=0$, where $\mathrm{v}$ - vector of velocity, $\mathrm{t}$ - time, $\mathrm{p}-$ pressure, $\rho$ - medium density, $\nabla p=$ $\operatorname{gradp}$.

If we apply the impulse model of problems of hydromechanics, then we can obtain the field velocity through potential.

$\bar{v}=\nabla\left(-\frac{P}{\rho}\right)=\nabla \varphi$, where $\varphi=-P / \rho-$ potential of speed, $P=\int_{0}^{t_{0}} p d t, \mathrm{P}-$ impulse of pressure, and for $\varphi$ we have $\Delta \varphi=0$ ( $\Delta$ - Laplace operator), i.e. $\varphi$ function is harmonic.

In the case when the velocity field is flat (tasks about the explosion of cord charges), the complex current potential $w(z)=\varphi(x, y)+i \psi(x, y)(\psi(x, y)-$ a current function complexly connected to $\varphi(x, y)$, which is an analytical function of the complex coordinate $z=x$, and its derivative has the form $\omega=\frac{d w}{d z}=v_{x}-i v_{y}=v e^{-i \theta}\left(v, \theta, v_{x}, v_{y^{-}}\right.$ correspondingly the value, argument and components of the velocity vector) and is called complex velocity.

There are different varieties of impulse-hydrodynamic model. The paper uses a solidliquid model, where the ground is described by the equations of ideal incompressible liquid, only in some area near the charge. Outside of this area, the soil behaves like an absolutely rigid body; the boundary separating the liquid is a solid wall, which is located from the condition that the velocity module on it is equal to a critical value. The critical velocity is a strength characteristic of the medium and is defined as follows. Fracture is considered to occur when the specific kinetic energy of the medium particles $e=\frac{\rho v^{2}}{2}$ exceeds the limit specific energy $e_{*}=\sigma_{*}^{2} /(2 E)$ required for the destruction of the medium (here $E-$ Young's module, $\sigma_{*}-$ yield strength limit). Then from the conditione $=e_{*}$ critical velocity can be found by formula $v^{*}=\sigma_{*} / \sqrt{\varrho E}$. The aim of the work is to develop a new method for calculating canals, trenches and sinkholes obtained by blasting. 


\section{Methods}

Explosive technologies are successfully used to create pits and trenches in transport construction. To solve these problems, methods of the theory of functions of a complex variable may be successfully used. Let us solve the Hilbert problem with discontinuous coefficients for a bipartite domain and apply the solution to the problem of explosion.

Let a rectangle $S^{+}$with vertices at points $0, \omega_{1}, \omega_{2}, \omega_{1}+i \omega_{2}, i \omega_{2}$, where $\omega_{1}>0, \omega_{2}>0$ are some real numbers be given in the plane $z=x+i y$. Denote by $l_{1}$ and $l_{1}^{\prime}$ respectively the lower and upper bases of the rectangle, and by $l_{2}$, the segment of the imaginary axis, for which $0 \leq y \leq \omega_{2}$. For the positive direction on the segment $l_{1}$ we take the direction from the origin to the point $\omega_{1}$, and on the segment $l_{1}^{\prime}$ we take the direction from the point $\omega_{1}+i \omega_{2}$ to the point $i \omega_{2}$.

It is required to find a function $F(z)=u(x, y)+i v(x, y)$, which is single-valued and analytic in $S^{+}$, periodic with period $\omega_{1}$, that is $F(t)=F\left(t+\omega_{1}\right), t \in l_{2}$, continuously continued on all points of the contour $L=l_{1}+l_{1}^{\prime}$, except maybe a finite number of given points $c_{k} k=\overline{1, N}$, lying on the bases of the rectangle, near which $|F(z)|<$ const $/\left|z-c_{k}\right|^{\lambda}, 0 \leq \lambda=$ const $<1$ by the boundary condition

$$
a(t) u(t)+b(t) v(t)=c(t), t \in L .
$$

Here $a(t), b(t), c(t),-$ the set on the real functions having discontinuities of the first kind at points $c_{k}$ and satisfying the Gölder condition on each of the segments (including the endpoints) into which the lines $l_{1}, l_{1}^{\prime}$, with points $c_{k}, k=\overline{1, N}$ are divided.

Without reducing generality, we may assume that $\lim _{t \rightarrow i \omega_{2}}[a(t)+i b(t)]=\lim _{t \rightarrow \omega_{1}+i \omega_{2}}[a(t)+i b(t)], t \in l_{1}^{\prime}$, $\lim _{t \rightarrow 0}[a(t)+i b(t)]=\lim _{t \rightarrow \omega_{1}}[a(t)+i b(t)], t \in l_{1}$.

We will solve the problem by the Muskhelishvili method, reducing it to the Riemann problem for a double-periodic function. Denote $S^{-}$by rectangle, symmetric about the $x$ axis, and introduce the function

$$
\Phi(z)=\left\{\begin{array}{l}
F(z), z \in S^{+} ; \\
\overline{F(\bar{z})}=\overline{F(z)}, z \in S^{-} .
\end{array}\right.
$$

This function is analytic in the regions $S^{+}$and $S^{-}$. Let us $\Phi(z)$ continue the function up and down periodically $\Phi\left(z+2 i \omega_{2} k\right)=\Phi(z)$ by assuming, where $k$ is an integer. Thus we obtain a bi-periodic function $\Phi(z)$ with periods $\omega_{1}, 2 i \omega_{2}$, which satisfies the symmetry condition, i.e. $\Phi(z)=\overline{\Phi(\overline{z)}}=\overline{\Phi(z)}, z \in S^{+}+S^{-}$.

The boundary condition (1) can be given the form

$$
\Phi^{+}(t)=G(t) \Phi^{-}(t)+g(t), t \in L
$$


where $G(t)=\frac{-(a(t)+i b(t))}{(a(t)-i b(t)}, g(t)=\frac{2 c(t)}{(a(t)-i b(t))}$, and, refers to $\Phi^{+}(t), \Phi^{-}(t)$ the values of the function at $z \rightarrow t \in L$ respectively left or right of $L$.

So we come to the Riemann problem for a bi-periodic function $\Phi(z)$, which must satisfy the condition .

$$
\Phi(z)=\overline{\Phi(\overline{z)}}=\overline{\Phi(z)}, z \in S^{+}+S^{-}
$$

Let us denote by

$\widetilde{\theta}=\arg (-G(t))=\arg \left((a(t)+i b(t) /(a(t)-i b(t)))=\widetilde{\theta}_{1}(t)+2 \pi \beta_{1}(t) n_{1}\right.$,

where $\beta_{1}(t)=1$, on $l_{1}^{\prime}, \beta_{1}(t)=0$ on $l_{1}, \widetilde{\theta}_{1}(t)$ is a definite branch of $\arg ((a(t)+i b(t) /(a(t)-i b(t)))$, continuous on each of the segments of, for which are divided $l_{1}, l_{1}^{\prime}$ with the points $c_{k}, n_{1}-$ is an integer, which will be defined below. As in ([10], pp. 269-270), $c_{k}$ we will call, in which $\widetilde{\alpha}_{k}=\widetilde{\theta}\left(c_{k}-0\right)-\widetilde{\theta}\left(c_{k}+0\right)=\widetilde{\theta}_{1}\left(c_{k}-0\right)-\widetilde{\theta}_{1}\left(c_{k}+0\right)=2 \pi n_{2}$, where $n_{2}$ is an integer, singular, and points $c_{k}$ in which $\widetilde{\alpha}_{k}=\widetilde{\theta}\left(c_{k}-0\right)-\widetilde{\theta}\left(c_{k}+0\right) \neq 2 \pi n_{2}$, where $k=\overline{1, N^{\prime}}$ is not singular.

We will look for the solution, bounded near the singular points $c_{k}, k=\overline{1, q}$, and unbounded near the remaining singular points $c_{k}, k=\overline{q+1, N^{\prime}}$. We choose the branch $\widetilde{\theta}_{1}=\arg \left((a(t)+i b(t) /(a(t)-i b(t)))\right.$ as follows. On the segment $l_{1}$ joining the points $z=0$ and $z=c_{k_{1}}\left(c_{k_{1}}-\right.$ the first of the discontinuity points on $l_{1}$, occurring when passing $l_{1}$ in the positive direction), and on the segment $l_{1}^{\prime}$ joining the points $z=i \omega_{2}+\omega_{1}$ and $z=c_{k_{1}^{\prime}}\left(c_{k_{1}^{\prime}}-\right.$ the first of the discontinuity points on $l_{1}^{\prime}$, occurring when passing $l_{1}^{\prime}$ in the positive direction), the branch value $\widetilde{\theta}_{1}(t)$ is taken arbitrarily. On other segments $l_{1}, l_{1}^{\prime}$, connecting two neighboring breakpoints, $\widetilde{\theta}_{1}(t)$ is chosen so that $0<\left(\widetilde{\theta}_{1}\left(c_{k}-0\right)-\widetilde{\theta}_{1}\left(c_{k}+0\right)\right) /(2 \pi)<1, k=\overline{1, q}$, $-1<\left(\widetilde{\theta}_{1}\left(c_{k}-0\right)-\widetilde{\theta}_{1}\left(c_{k}+0\right)\right) / 2 \pi<0, k=\overline{q+1, N^{\prime}}$, $\widetilde{\theta}_{1}\left(c_{k}-0\right)-\widetilde{\theta}_{1}\left(c_{k}+0\right)=0, k=\overline{N^{\prime}+1, N}$.

Let us introduce integers $\chi_{1}^{\prime}=\left(\widetilde{\theta}\left(\omega_{1}-0\right)-\widetilde{\theta}(0+0)\right) /(2 \pi)=\left(\widetilde{\theta}_{1}\left(\omega_{1}-0\right)-\widetilde{\theta}_{1}(0+0)\right) /(2 \pi)$, $\chi_{2}^{\prime}=\left(\widetilde{\theta}\left(i \omega_{2}-0\right)-\widetilde{\theta}\left(\omega_{1}+i \omega_{2}+0\right)\right) /(2 \pi)=\left(\widetilde{\theta}_{1}\left(i \omega_{2}-0\right)-\widetilde{\theta}_{1}\left(\omega_{1}+i \omega_{2}+0\right)\right) /(2 \pi)$ and call the number $\chi^{\prime}=\chi_{1}^{\prime}+\chi_{2}^{\prime}$ the index of the Hilbert problem of the class of solutions under consideration.

I. Let $\chi_{1}^{\prime}$ and $\chi_{2}^{\prime}$ are odd numbers, i.e. $\chi_{1}^{\prime}=2 \chi_{1}-1, \chi_{2}^{\prime}=2 \chi_{2}-1$, where $\chi_{1}, \chi_{2}$ are integers. Take an arbitrary fixed point $z_{2}\left(0<\operatorname{Im} z_{2}<\omega_{2}\right)$ and construct the function $X(z)=\exp (\Gamma(z))[\sigma(z)]^{-2 \chi_{1}}\left[\sigma\left(z-i \omega_{2}\right) \sigma\left(z+i \omega_{2}\right)\right]^{-\chi_{2}}\left[\sigma\left(z-z_{2}\right) \sigma\left(z-\overline{z_{2}}\right)\right]^{\chi_{1}+\chi_{2}}$, 
Where

$$
\Gamma(z)=\frac{1}{2 \pi} \int_{L}[\pi+\widetilde{\theta}(t)] \varsigma(t-z) d t-\eta_{2} \frac{1}{4 \pi} \int_{L}[\pi+\widetilde{\theta}(t] d t,
$$

$\varsigma(t-z), \sigma(z)$ are, respectively, the Weirstrass zeta and sigma functions for periods $\omega_{1}$ and $2 i \omega_{2}, \eta_{2}=2 \varsigma\left(i \omega_{2}\right)$. The function will be biperiodic if

$$
\alpha_{1}+2\left(\chi_{1}+\chi_{2}\right) \operatorname{Re} z_{2}=0
$$

Here

$$
\alpha_{1}=\frac{1}{2 \pi} \int_{L}[\pi+\widetilde{\theta}(t)] d t=\frac{1}{2 \pi} \int_{L}\left[\pi+\widetilde{\theta}_{1}(t)\right] d t-n_{1} \omega_{1},
$$

and let us agree to choose $n_{1}$ so that $0 \leq \alpha_{1}<\omega_{1}$. It is clear that such a choice is always possible. The fulfillment of relation (7) can be achieved by choosing $\operatorname{Re} z_{2}$, if $\chi_{1}+\chi_{2} \neq 0$. It is easy to see that $\overline{X(z)}=X(z)$.

Taking into account the results of Muskhelishvili ([23], pp. 53, 54, 75) we can show that the function $X(z)$ will have poles of the first order at the points $z=0, z=i \omega_{2}$ turns to zero of the order $\tilde{\alpha}_{k}$ at points $c_{k}$ at which $0<\widetilde{\alpha}_{k}<1$; turns to infinity of integrable order $-\widetilde{\alpha}_{k}$ at points $c_{k}$ for which $-1<\tilde{\alpha}_{k}<0$; at all other points of $L$ function is nonzero and satisfies the Gölder condition.

In the case under consideration $\chi^{\prime}=2\left(\chi_{1}+\chi_{2}\right)-2=2 \chi-2$ is an even number, here $\chi=\chi_{1}+\chi_{2}$.

a). Let that $\operatorname{be}\left(\chi^{\prime}+2\right) / 2=\chi=\chi_{1}+\chi_{2}<0$, i.e. $\chi^{\prime}<-2$. The fulfillment of condition (7) can be achieved by selecting $\operatorname{Re}\left(z_{2}\right)$ ( $\operatorname{Im} z_{2}$ remains an arbitrary). According to (5), the function $X(z)$ will have order $-\chi$ at points $z_{2}, \overline{z_{2}}$ and first-order poles at points $z=0, z=i \omega_{2}$.

By analogy to what was done in ([23], p. 146), it can be shown that the function

$$
F(z)=(\Phi(z)+\overline{\Phi(z)}) / 2=X(z)\left\{\frac{1}{\pi i} \int_{L} \frac{c(t) \varsigma(t-z) d t}{X^{+}(t)[a(t)-i b(t)]}+\eta_{2} \frac{1}{2 \pi i} \int_{l_{1}^{\prime}} \frac{c(t) d t}{\overline{X^{+}(t)}[a(t)+i b(t)]}+\operatorname{Re} C\right.
$$

is a solution of the Hilbert problem under study and will be bi-periodic if [24]:

$$
\int_{L} \frac{c(t) d t}{X^{+}(t)[a(t)-i b(t)]}=0
$$

Here $\mathrm{C}$ is an arbitrary complex constant. Function (8) will be analytic in the domain $S^{+}$if the expression in curly brackets of the right-hand side of formula (8) is zero at points $0, i \omega_{2}$ and at a point $z_{2}$ has zero of order $-\chi$. Consequently, the following conditions must be satisfied:

$$
\begin{aligned}
& \frac{1}{\pi i} \int_{L} \frac{c(t) \varsigma(t) d t}{X^{+}(t)[a(t)-i b(t)]}+A^{*}+\operatorname{Re} C=0 \\
& \frac{1}{\pi i} \int_{L} \frac{c(t) \varsigma\left(t-i \omega_{2}\right) d t}{X^{+}(t)[a(t)-i b(t)]}+A^{*}+\operatorname{Re} C=0
\end{aligned}
$$




$$
\begin{aligned}
& \frac{1}{\pi i} \int_{L} \frac{c(t) \varsigma\left(t-z_{2}\right) d t}{X^{+}(t)[a(t)-i b(t)]}+A^{*}+\operatorname{Re} C=0 \\
& \frac{1}{\pi i} \int_{L} \frac{c(t) \varsigma^{(k)}\left(t-z_{2}\right) d t}{X^{+}(t)[a(t)-i b(t)]}=0, k=\overline{1,-\chi-1 .}
\end{aligned}
$$

Here we assume

$$
A^{*}=\eta_{2} \frac{1}{2 \pi i} \int_{L} \frac{c(t) d t}{\overline{X^{+}(t)}[a(t)-i b(t)]} .
$$

In addition to these conditions, we must also satisfy (9), which is, as is easily seen, a valid condition. It is also easy to check that each of the conditions (10) and (11) are real.

It is clear from the above that the problem under consideration is solvable if conditions (9) and (10)-(13) are satisfied. By singling out the real and imaginary parts in (12) and (13), we arrive at $-2 \chi$ the real relations. One of the conditions (10), (11) can be satisfied by selecting $\operatorname{Re} C$. Thus, the problem under study is solvable if $-\chi^{\prime}$ the real solvability conditions are satisfied. In this case it has a single solution defined by formula (8).

The other cases are treated similarly.

As an example of the application of the above results, let us consider the problem of the explosion of an infinitely long buried charge within the framework of the solid-liquid model. The motion is assumed to be plane-parallel, so it is sufficient to study it in the plane perpendicular to the axis of the cord charge, and take this plane as the plane of the complex variable $z=x+i y$. Let's direct the real axis along the plane coinciding with the ground surface, the charge will be considered to be located in the half-plane of $y<0$. The fluid flow is described by the complex potential $w=w(z)=\varphi+i \psi$. Let the charge in the lower half-plane occupy the position $A B$, which makes an angle $\alpha_{0}$ with the axis $x$. Denote the flow area by $D_{z}$. The flow area is bounded from above by the segment $Q C$ of the real axis and the abscissa of these points satisfy the condition $x_{Q}<x_{C}\left(x_{Q}=0\right)$, and from below it is bounded by the line $Q H C$, which is a current line with a branch point $H$ besides on the line $Q H C$ the velocity remains constant and equal to the critical $v_{*}$.

Let $\omega=w^{\prime}(z)=v \exp (-i \theta)=v_{x}-i v_{y}$ be the complex velocity of the flow. At the boundary of the charge and at the section of $Q C$ the velocity potential is $\varphi=c o n s t$. On them, the velocity vector $v \exp (i \theta)$ is perpendicular to the specified sections. In the region $D_{z}$, draw a section along the current line from the branching point $H$ of the flow. Let $H^{-} L_{1}$ and $H^{=} L_{2}$, respectively, be the left and right sides of the slit, and $\Gamma_{z}$ be the boundary of the region $D_{z}$. At the boundary segments $\Gamma_{z}$ we have the following boundary conditions for the function $w(z): \varphi=0$ at $Q C \varphi=-\varphi_{0}\left(\varphi_{0}>0\right)$ at $L_{1} A B L_{2}$, where $-\varphi_{0}$ is the value of the potential on the charge, $\psi=0$ at $Q H^{-} L_{1}, \psi=\psi_{0} L_{2} H^{+} C$ (the value $\psi_{0}$ is given). The velocity $v_{\text {on }} \mathrm{QH}^{-}, \mathrm{H}^{+} \mathrm{C}$ is constant and equal to the critical velocity $v_{*}$. 
Let us introduce dimensionless variables according to the relations $w^{\prime}=\frac{w}{\varphi_{0}}, v^{\prime}=\frac{v}{v_{*}}, z^{\prime}=\frac{z v_{0}}{\varphi_{0}}$. So, on the rectilinear sections of the boundary $\Gamma_{z}$ the real part of the function $w(z)$ is given, on the curvilinear sections - the imaginary part of the function $w(z)$, besides on the lines $Q H^{-}, H^{+} C|d w / d z|=1$. We need to determine the line $Q H C$, as well as the position and dimensions of the charge. The area of complex potential is a rectangle with vertices at points $-1,-1+i \psi_{0}^{\prime}, i \psi_{0}^{\prime}, 0$. The solution of the problem is sought in parametric form. For this purpose, a rectangle $D_{u}$ with vertices $2 \omega_{1}, 2 \omega_{1}+i \omega_{2}, i \omega_{2}, 0$ at points in the plane of the auxiliary complex variable $u=u_{1}+i u_{2}\left(\omega_{1}>0, \omega_{2}>0\right)$ is introduced. The quantities $\omega_{1}, \omega_{2}$ are arbitrary constants, and the points $H^{+}$correspond to $u=0, H^{-}$$2 \omega_{1}, L_{2}-i \omega_{2}, L_{1}-2 \omega_{1}+i \omega_{2}$. The function mapping conformally the domain $D_{u}$ on the domain $D_{w}$ has form

$$
w(u)=\frac{i \psi_{0}^{\prime}}{2 K(k)} \int_{\omega^{*}}^{u} \frac{d n\left[-K^{*}\left(u-\omega^{*}\right), \lambda\right] d u}{\sqrt{1-k^{2} s n^{2}\left[-K^{*}\left(u-\omega^{*}\right), \lambda\right]}}+i \frac{\psi_{0}^{\prime}}{2}-1,
$$

where $\operatorname{dn}(u, \lambda)$ is the elliptic Jacobi function and $\omega^{*}=\omega_{1}+i \omega_{2}, K^{*}=K^{\prime}(\lambda) / \omega_{2}$. Wherein $K(k)$ is a complete elliptic integral of the first kind, and the parameter $k$ is determined from the relation $K^{\prime}(k) / K(k)=2 / \psi_{0}^{\prime}$, where $K^{\prime}(k)=K\left(k^{\prime}\right), k^{\prime}=\sqrt{1-k^{2}}$.

Let us introduce the analytic in the $D_{u}$ function $X_{1}(u)=\ln \frac{d w}{d z}+i \frac{\pi u}{\omega_{1}}$, satisfying the periodicity condition $X_{1}\left(i u_{2}+2 \omega_{1}\right)=X_{1}\left(i u_{2}\right)$, where $u_{2}$ is the real variable. To define $X_{1}(u)$, analytic in the rectangle, we have a Hilbert boundary problem with discontinuous coefficients

$$
a(t) \operatorname{Re} X_{1}(t)+b(t) \operatorname{Im} X_{1}(t)=c(t), t \in L
$$

where

$$
\begin{aligned}
& a(t)=1, b(t)=0, c(t)=0 \text { on } H^{+} C, Q H^{-}, \\
& a(t)=0, b(t)=1, c(t)=-\frac{\pi}{2}+\frac{\pi u_{1}}{\omega_{1}} \text { on } C Q, \\
& a(t)=0, b(t)=1, c(t)=-\frac{3 \pi}{2}+\frac{\pi u_{1}}{\omega_{1}}-\alpha_{0} \text { on } L_{1} A, \\
& a(t)=0, b(t)=1, c(t)=-\frac{\pi}{2}+\frac{\pi u_{1}}{\omega_{1}}-\alpha_{0} \text { on } A B
\end{aligned}
$$




$$
a(t)=0, b(t)=1, c(t)=\frac{\pi}{2}+\frac{\pi u_{1}}{\omega_{1}}-\alpha_{0} \text { on } B L_{2} \text {. }
$$

It is clear that we have to look for a solution of the Hilbert problem, bounded near nonseparate points. Accordingly, let us assume $\theta_{1}(t)=0$ at $H^{+} C, \theta_{1}(t)=-\pi$ at $C Q, \theta_{1}(t)=-2 \pi, Q H^{-}, \theta_{1}(t)=-\pi$ where $\theta_{1}(t)$ denotes the same as in (I) of this paper. The index of the Hilbert problem is $\chi^{\prime}=\chi_{1}^{\prime}+\chi_{2}^{\prime}=-1$, where $\chi_{1}^{\prime}=-1, \chi_{2}^{\prime}=0$. In this case, the solution of the Hilbert problem is defined with exactness up to a real arbitrary constant term by the formula

$$
X_{1}(u)=X_{2}(u)\left\{\frac{1}{\pi i} \int_{L} \frac{c(t) \varsigma(t-u) d t}{X_{2}^{+}(t)[a(t)-i b(t)]}-\eta_{2} \frac{1}{2 \pi i} \int_{l_{1}} \frac{c(t) d t}{X_{2}^{+}(t)[a(t)-i b(t)}\right\}+C^{\prime},
$$

where $C^{\prime}$ is an arbitrary real constant. The function $X_{2}(u)$ is expressed by the formula $X_{2}(u)=\left[\sigma\left(u-\widetilde{c}^{\prime}\right) \sigma(u-q)\right]^{1 / 2}\left[\sigma(u) \sigma\left(u-2 \omega_{1}\right)\right]^{-1 / 2}$. The constants $\widetilde{c}^{\prime}, q$ are defined by finding the function $w(u)$. The function conformally mapping the domain $D_{u}$ to the domain $D_{z}$ has the form

$$
z(u)=\int_{q}^{u} w^{\prime}(u) \exp \left(-X_{1}(u)+i \pi u\right) d u \text {. }
$$

Using this formula it is possible to obtain all mechanical and geometric parameters of the explosion. Table 1 shows the results of calculations to determine the geometric parameters of trenches.

Table 1. Geometric parameters of trenches.

\begin{tabular}{|c|c|c|c|c|}
\hline $\begin{array}{c}\text { example } \\
\text { number }\end{array}$ & 1 & 2 & 3 & 4 \\
\hline$\alpha_{0}$ & 0.1493 & 0.5799 & -0.3170 & -0.0009 \\
\hline$x_{H}$ & 0.4836 & 0.4760 & 0.4919 & 0.4864 \\
\hline$y_{H}$ & -0.3175 & -0.3359 & -0.3205 & -0.3091 \\
\hline$x_{A}$ & 0.4225 & 0.4678 & 0.4245 & 0.4218 \\
\hline$y_{A}$ & -0.2356 & -0.2803 & -0.2067 & -0.2180 \\
\hline$x_{B}$ & 0.5505 & 0.5781 & 0.5478 & 0.5511 \\
\hline$y_{B}$ & -0.2163 & -0.2080 & -0.2472 & -0.2181 \\
\hline$x_{C}$ & 0.9725 & 0.9708 & 0.9722 & 0.9728 \\
\hline
\end{tabular}

\section{Results and discussion}

The use of force is widespread in building technologies [25]. In this work, the methods of the theory of analytical functions in the framework of the solid-liquid model were used to solve the problem of determining the excavation of the ejection during the explosion of a buried charge. Methods of the theory of analytical functions are widely used in practice [22, 26-29]. Due to the importance of boundary value problems, research is conducted in the 
direction of solving Hilbert, Riemann boundary value problems with an infinite index [30,31], and also solutions in the case of special behavior of the boundary value coefficients [32]. In these works the behavior of singular integrals at special points [33] and peculiarities of application of the Christoffel-Schwarz formula [34] are used.

In the present work the boundary Hilbert problem with discontinuous coefficients for a bipartite domain was solved, which allowed to carry out a complete analysis of the buried charge explosion problem, to determine the boundary of the ejection notch, as well as to find the coordinates of important geometric characteristics of the notch.

\section{Conclusion}

In the process of construction of road embankments, canals and foundation pits there is a problem of moving some soil mass in a given direction and calculating the geometry of channels and foundation pits. To calculate the ejection of a buried cord charge we propose a method that uses the solution of the boundary Hilbert problem with discontinuous coefficients for a bipartite domain. The solution is obtained in this study.

In the study, calculated schemes are established and simulation of the process of formation of the ditch (trench, channel) is carried out, geometric and physical parameters affecting the shape and size of the ditch are determined, formulas for finding in the physical plane of all the interesting features of the ditch are obtained, numerical calculations have been performed.

\section{References}

1. V. M. Fomina, B. V. Postinkova, B. Kolotilova, V. S. Shalaev, Yu. V. Shalaev, N. F. Floryac, Modeling Shock Wave Processes in a Mine Opening with Permeable Barriers, Journal of Mining Science 55(1), 67-85 (2019) DOI: 10.1134/S106273911901524X

2. V. N. Tyupin, Intensification of heap leaching of ores using energy of explosion, Mining Journal, 8, 78-84 (2019)

3. E. O. Tarasenko, V. S. Tarasenko, A. V. Gladkov. Mathematical Modeling of Sedimentary Loess Soils Compaction in the North Caucasus by Deep Explosions, Izvestija Tomskogo Polytechnicheskogo Universiteta, 330 (11), 34-46 (2019)

4. Morteza Esmaieili, Boheria Tavakovi, Numerical evaluation of square arrangement of charges in explosive compaction, Soil Dynamics and Earthquake Engineering, 130, 106001, 1-14 (2020)

5. E. N. Sher, Development of a System of Plane Radial Cracks in Explosion of Linear Blasthole and Borehole and Burning Charges, Applied mechanics and technical physics, 58(5), 201207 (2017)

6. E. N. Sher, Shape and size of radial cracks under blasting of two closely spaced blastholecharges, Journal fundamental and applied mining questions, 1(13), 250-255 (2016)

7. B. Liang, H. Jang, T. Xu, Impact dynamic response of near-field explosion in buried gas pipeline based on SPH-FEM coupling algorithm, ActaPetroleiSinic, 38(11), 13261334 (2017)

8. Chen Jian-Yu, Lien Fue-sang, Simulations for soil explosion and its effects on structures using SPH method, International Journal of Impact Engineering, 112, 15 21 (2017) DOI: 10.1016/j.ijimpeng.2017.10.008

9. Alexander I. Lopato, Pavel S. Utkin, The usage of grid-characteristic method for the simulation of flows with detonation waves, Smart Modeling for Engineering Systems, in Proc. of the Conf. 50 Years of the Development of Grid-Characteristic Method, 
Moscow, March 31-April 3 2018, Smart Innovation, Systems and Technologies, 133, 281-290 (2019)

10. J. Fang, Yu. Yao, A. A. Zheltovodov, L. Lu, Study of three-dimensional interaction shock wave, Turbulent boundary layer initiated by a single fin AIAA Journal, 55 (2), 509-523 (2017)

11. M. Yu. Timokhin, H. H. Struchtrup, A. A. Kohanchik, E. A. Bondar, Various variants of the equations of moment R13 applied to the shock-wave structure, Physics of fluids, 29 (3), 37-45 (2017)

12. M. Yu. Timokhin, H. H. Struchtrup, A. A. Kohanchik, E. A. Bondar, Note from the publisher: Various variants of the equations of the moment R13 applied to the shockwave structure, Journal Physics of liquids, 29 (4), 499-501 (2017)

13. P. Bulat, Shock-Wave processes and shock-Wave structures, Journal Akustika, 27(1), 12-15 (2017)

14. A. A. Sysoev, Analysis of the borehole charge initiation systems at the open-cast mines (in Russian), .Izvestia of higher educational institutions. Mining journal, 4, 60-67 (2016)

15. Chen Shuxing, L. I. Dening, Conical shock waves in supersonic flow, Journal of Differential Equations, 1, 45-61 (2020)

16. A. I. Lopato, P. S. Utkin, Mathematical modeling of detonation initiation and propagation in the complex-shaped domains, in Proc. XXXIII Int. Conf. on Equations of State for Matter, Elbrus, Kabardino-Balkaria, Russia, March 1-6, 2018, JPCS, 1147 (2019)

17. M. Yu. Timokhin, Ye. A. Bondar, A. A. Kokhanchik, M. S. Ivanov, I. E. Ivanov, I. A. Kryukov, Physics of Fluids (American Institute of Physics United States.AIP Publishing LLC, 2015)

18. P. S. Utkin, Numerical simulation of shock wave - dense particles cloud interaction using Godunov solver for Baer-Nunziato equations, International Journal of Numerical Methods for Heat \& Fluid Flow, 3225-3241 (2019)

19. A. T. Basilevsky, L. B. Ronca, B. A. Ivanov, On the rate of ancient meteoroidal flux: studies of the premare crater population on the Moon, Moon Planets, 23 (3), 355-371 (1980)

20. V. V. Selivanov, S. V. Fedorov, Ya. M. Nikolskaya, S. V. Ladov, Research of the explosive formation of a compact element for meteoroids fragments and space debris modeling, Acta Astronautica, 163, 84-90 (2019)

21. V. V. Selivanov, S. V. Fedorov, Ya. M. Nikolskaya, S. V. Ladov, Compact element formation for the modeling of the high-velocity impacts of particles onto spacecraft materials and construction elements in earth conditions, Acta Astronautica, 135, 34-43 (2016)

22. V. M. Kuznetsov, Mathematical models of the explosive case (Publishing House Science, Novosibirsk, Russia, 260, 1977)

23. N. I. Muskhelishvili, Singular integral equations. Boundary Problems of the Theory of Functions and Some of Their Applications to Mathematical Physics (Fizmatgiz, Moscow, 599, 1962)

24. W. T. Koiter. Some general theorems on doubly-periodic and quasi-periodic functions Proc. Konikl. Nederl. akad. wet., A-62, 2, 120-128 (1959)

25. A. G. Mudrov, A. A. Mudrova, Use of regulated force effect in building technologies, Izvestija KGASU, 4(50), 410-417 (2019)

26. S. L. Tolokonnikov, The shape of the crater formed by an explosive wedge-shaped cord charge on the ground surface, Journal Moscow University Mechanics Bulletin, 73 ( 6), 149-152 (2018) 
27. R. B. Salimov, The solution of the inverse edge problem of aerohydrodynamics in a new formulation, Izvestia vuzov.Mathematics, 9, 96-101 (2017)

28. R. B. Salimov. Solution of the inverse mixed boundary task of aerohydrodynamics in a new formulation, Izvestia vuzov. Mathematics, 5, 86-92 (2018)

29. V. I. Bezrodnykh, S. I. Vlasov, Asymptotics of the Riemann-Hilbert Problem for a Magnetic Reconnection Model in Plasma, Computational Mathematics and Mathematical Physics, 60, 11, 1898-1914 (2020) DOI: 10.1134/S0965542520110056

30. R. B. Salimov, P. L. Shabalin, About solvability of a homogeneous Hilbert problem with discontinuities of coefficients and bilateral whirling on infinity of logarithmic order, Izvestia vuzov. Mathematics, 1, 36-48 (2016)

31. R. B. Salimov, A. Z. Suleymanov, New approach to the solution of the homogeneous edge Roman's problem on the beam with infinite index, Izvestia vuzov. Mathematics, $\mathbf{5}$, 71-76 (2017)

32. P. L. Shabalin, A. Kh. Fatykhov, Inhomogeneous Hilbert Boundary Value Problem with Several Points of Logarithmic Turbulence, Russian Mathematics, 65, 1, $57-71$ (2021) DOI: $10.3103 / \mathrm{S} 1066369 \mathrm{X} 21010059$

33. R. B. Salimov, New asymptotic representation of the singular integral with the Hilbert nucleus near the point of weak density continuum, Izvestia vuzov. Mathematics, 4, 7378 (2016)

34. P. L. Shabalin, E. N. Karabasheva, On univalent mappings performed by the generalized Christoffel-Schwarz formula, Journal of Mathematical Sciences, 245(1), 8388 (2020) DOI:10.1007/s10958-020-04678-4 